\title{
The effectiveness of the Mclsaac clinical decision rule in the management of sore throat: an evaluation from a pediatrics ward
}

\author{
Saravanapriya Thillaivanam¹, Arwa M. Amin², Sheila Gopalakrishnan ${ }^{3}$ and Baharudin Ibrahim
}

BACKGROUND: Sore throats may be due to either viral or group A beta hemolytic streptococcus (GABHS) infections; but diagnosis of the etiology of a sore throat is difficult, often leading to unnecessary antibiotic prescriptions and consequent increases in bacterial resistance. Scoring symptoms using the Mclsaac clinical decision rule can help physicians to diagnose and manage streptococcal infections leading to sore throat and have been recommended by the Ministry of Health, Malaysia. In this paper, we offer the first assessment of the effectiveness of the Mclsaac rule in a clinical setting in Malaysia.

METHOD: This study is a retrospective review of 116 pediatric patients presenting with sore throat. Group A comprised patients before the implementation of the Mclsaac rule and Group B comprised patients after the implementation.

RESULTS: Unnecessary throat swab cultures were reduced by $40 \%(P=0.003)$. Redundant antibiotic prescriptions were reduced by $26.5 \%(P=0.003)$ and the overall use of antibiotics was reduced by $22.1 \%(P=0.003)$. The pediatricians' compliance rate to Mclsaac rule criteria was $45 \%$ before implementation of the Mclsaac rule, but improved to $67.9 \%(P=0.0005)$ after implementation.

DISCUSSION: The Mclsaac rule is an effective tool for the management of sore throat in children in Malaysia.

$S_{t}^{o}$ ore throat is one of the most common upper respiratory tract infections (URTIs) presented to pediatric clinics (1). It can be caused by either viral or streptococcal infections (2), but it has been reported that viral causes of sore throat represent $>80 \%$ of the cases, while Group A beta hemolytic streptococcus (GABHS) is responsible for $15 \%(2-4)$. It is difficult, however, to discriminate between viral and GABHS infections of sore throat by means of clinical signs and symptoms, and this leads to a high rate of inaccurate diagnoses $(5,6)$. In this context, and since GABHS infection is associated with severe complications, such as acute rheumatic fever, peritonsillar abscess, and rheumatic disease, which may in severe cases lead to death (7), clinical practitioners often take a precautionary approach by prescribing antibiotics immediately after presentation with sore throat. Nevertheless, a Cochrane review on the delayed use of antibiotics for respiratory infections indicated that there was no difference in complication rates whether the use of antibiotics was immediate or delayed (8). Furthermore, it has been documented that the overuse of antibiotics in URTIs is associated with increased bacterial resistance and adverse drug reactions (9). Such microbial resistance places a great burden on the health cost and on public health generally (10), and, therefore, a judicious approach to the prescription of antibiotics in cases of sore throat is imperative to prevent bacterial resistance.

The World Health Organization had classified two criteria for the diagnosis of streptococcal sore throat in children: tender, enlarged lymph node on the neck and white exudates on the throat (11). These two criteria are considered to be an indication for the use of antibiotics. This approach has higher specificity but lower sensitivity (12). Accordingly, several clinical decision prediction rules have been developed to assist better management of sore throat and to prevent misuse of antibiotics. One such rule is the Centor Strep Score for adults, which was later modified to develop the McIsaac decision rule for children (13). The McIsaac clinical decision rule is a validated score tool which aims to reduce the redundancy of antibiotic use and improve the accuracy of GABHS diagnosis in the case of sore throat. The score predicts GABHS infection using five criteria: age, tonsillar swelling or exudates, anterior cervical lymphadenopathy, absence of cough, and temperature $>38^{\circ} \mathrm{C}$ (Table 1). The likelihood of patient having GABHS infection increases with the total score, which will be used to assist decisions in respect to antibiotic prescribing (13). Tables 1 and 2 show the McIsaac decision rule criteria and the suggested management according to the total score. The management of sore throat according to the McIsaac Score has been shown to result in a $48 \%$ reduction in antibiotic use, a $63.7 \%$ reduction in unnecessary antibiotic prescriptions, and a $35.8 \%$ reduction in the culture of throat samples (13).

'Department of Pharmacy, Hospital Kulim, Kulim, Kedah, Malaysia; ${ }^{2}$ Discipline of Clinical Pharmacy, School of Pharmaceutical Sciences, Universiti Sains Malaysia (USM), Minden, Pulau Pinang, Malaysia; ${ }^{3}$ Department of Pediatrics, Hospital Seri Manjung, Seri Manjung, Perak, Malaysia; ${ }^{4}$ Discipline of Clinical Pharmacy, School of Pharmaceutical Sciences, Universiti Sains Malaysia (USM), Minden, Pulau Pinang, Malaysia. Correspondence: Baharudin Ibrahim (baharudin.ibrahim@usm.my)

Received 9 March 2016; accepted 1 April 2016; advance online publication 22 June 2016. doi:10.1038/pr.2016.113 
Table 1. Mclsaac decision rule

\begin{tabular}{lc}
\hline Criteria & Points \\
\hline Temperature $>38^{\circ} \mathrm{C}$ & 1 \\
No cough & 1 \\
Tender anterior cervical adenopathy & 1 \\
Tonsillar swelling or exudates & 1 \\
Age 3-14y & 1 \\
Age 15-44y & 0 \\
Age $\geq 45 y$ & -1 \\
Total score & \\
\hline
\end{tabular}

Adapted with permission from ref. 13 .

Table 2. Mclsaac's suggested management according to the total score

\begin{tabular}{lcl}
\hline Total score & $\begin{array}{c}\text { Likelihood of } \\
\text { GABHS (\%) }\end{array}$ & \multicolumn{1}{c}{ Suggested management } \\
\hline 0 & $2-3$ & No culture or antibiotic is required \\
1 & $4-6$ & \\
2 & $10-12$ & $\begin{array}{l}\text { Culture all; treat only if culture } \\
\text { results are positive }\end{array}$ \\
3 & $27-28$ & $\begin{array}{l}\text { Treat with antibiotics on clinical } \\
\text { grounds, without culture }\end{array}$ \\
4 or 5 & $38-63$ &
\end{tabular}

GABHS, group A beta hemolytic streptococcus.

Adapted with permission from ref. 13 .

The Ministry of Health in Malaysia has recommended that the clinician's decision for sore throat management should be based on the McIsaac clinical decision rule. In practice, however, some clinicians do not apply this scoring mechanism, possibly due to the lack of data on its effectiveness in Malaysia, where GABHS infections have been reported to be especially prevalent in sore throat cases (as with other countries in tropical regions) (14). To date, the effectiveness of this score has not been evaluated in Malaysia, and therefore, this study aimed to evaluate the effectiveness of McIsaac scoring in reducing inappropriate use of antibiotics and in reducing unneeded throat swab cultures in children diagnosed with sore throat in Hospital Kulim, Malaysia. It also aimed to evaluate the compliance to the McIsaac rule among pediatricians in this hospital. This will shed light on the effectiveness of using this rule and will enable further improvements to clinical practice and public health in Malaysia.

\section{RESULTS}

The total bed head tickets with an initial diagnosis of sore throat which were reviewed were 116. Sixty of these were for Group A (July to September 2012) and 56 were in Group B (October to December 2012). In Group A, 53.3\% (32/60) were females while $50 \%$ (28/56) of Group B were females. There was no significant difference in gender between the two groups $(P=0.721)$. The means $(\mathrm{SD})$ of patient age were $4.1(2.8)$ and 3.4 (3.03) for Group A and B, respectively. This difference in
Table 3. Demographics and clinical data of the two groups ( $A$ and $B$ )

\begin{tabular}{llccl}
\hline \multirow{2}{*}{$\begin{array}{l}\text { Demographics/ } \\
\text { clinical data }\end{array}$} & \multicolumn{1}{c}{ Categories } & Group A & Group B & \\
\cline { 3 - 4 } Age & Mean (SD) & $4.12(2.8)$ & $3.38(3.03)$ & $0.039^{*}$ \\
Gender & Male & $28(46.7 \%)$ & $28(50 \%)$ & 0.721 \\
& Female & $32(53.3 \%)$ & $28(50 \%)$ & \\
Ethnicity & Malay & $50(83.3 \%)$ & $46(82.1 \%)$ & 0.871 \\
& Chinese & $3(5 \%)$ & $3(5.4 \%)$ & \\
& Indian & $7(11.7 \%)$ & $7(12.5 \%)$ & \\
Related medical & Allergic rhinitis & $2(3.3 \%)$ & $2(3.6 \%)$ & 0.667 \\
history & Bronchial asthma & $8(13.3 \%)$ & $8(14.3 \%)$ & \\
& Dermatitis & $1(1.7 \%)$ & $2(3.6 \%)$ & \\
& Having no other & $49(81.7 \%)$ & $44(78.6 \%)$ & \\
& illness & & & \\
& & & &
\end{tabular}

*There was a significant difference in age distribution among both the groups.

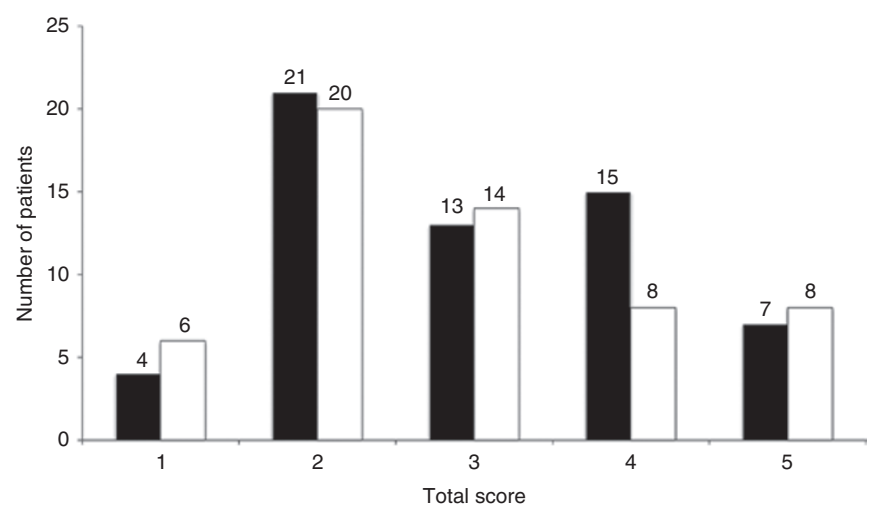

Figure 1. Patient distribution for every total score. The figure shows the frequency of Group A (black) and Group B (white) tickets in each score. Score 2 had the highest frequency in both groups.

age between the two groups was significant $(P=0.039)$. The demographics and clinical data for the two groups are detailed in Table 3. The highest total score among the two groups was Score 2, representing 35\% of Group A and 35.7\% of Group B. Figure 1 shows the distribution of the total score among the two groups.

There was no significant difference in the total number of cultures performed before and after the enforcement of the McIsaac decision rule $(P=0.787)$. For those with Score 2 , none of those cultured in Group A were positive for GABHS 0/4 (0\%), and only 2 out of 9 (22.2\%) of Group B were GABHS positive. Of the total cultures done in Group A, 40\% (8/20) were found not to comply with McIsaac's recommendations. All cultures in Group B, however, complied with McIsaac's suggestions and Group B also had a significantly lower incidence of redundant throat culture than Group A $(P=0.003)$. Table 4 details the throat swab culture rate against the total score in the two groups.

Almost 80\% (92/116) of the total population in this study were prescribed an antibiotic, but only $40.5 \%$ (47/116) were prescribed antibiotics as per McIsaac's suggestions. There 
Articles | Thillavanam etal.

Table 4. Throat swab culture rate

\begin{tabular}{|c|c|c|c|c|c|c|c|c|}
\hline $\begin{array}{l}\text { Total } \\
\text { score }\end{array}$ & $\begin{array}{l}\text { Number of } \\
\text { cultures from } \\
\text { Group A }\end{array}$ & $\begin{array}{c}\text { Number of } \\
\text { positive GABHS }\end{array}$ & $\begin{array}{l}\text { Number of } \\
\text { cultures from } \\
\text { Group B }\end{array}$ & $\begin{array}{c}\text { Number of } \\
\text { positive GABHS }\end{array}$ & $\begin{array}{c}P \\
\text { value }\end{array}$ & $\begin{array}{c}\text { Number of } \\
\text { redundant cultures } \\
\text { from Group A }(n=20)\end{array}$ & $\begin{array}{c}\text { Number of } \\
\text { redundant cultures } \\
\text { from Group B }(n=22)\end{array}$ & $P$ value \\
\hline 1 & $0(n=4)$ & $0(0 \%)(n=0)$ & $0(n=6)$ & $0(0 \%)(n=0)$ & 1 & $0(0 \%)(n=4)$ & $0(0 \%)(n=6)$ & 1 \\
\hline 2 & $4(n=21)$ & $0(0 \%)(n=4)$ & $9(n=20)$ & $2(22.2 \%)(n=9)$ & 0.074 & $0(0 \%)(n=21)$ & $0(0 \%)(n=20)$ & 1 \\
\hline 3 & $8(n=13)$ & $2(25 \%)(n=8)$ & $11(n=14)$ & $5(45.5 \%)(n=11)$ & 0.333 & $0(0 \%)(n=13)$ & $0(0 \%)(n=14)$ & 1 \\
\hline 4 & $5(n=15)$ & $5(100 \%)(n=5)$ & $0(n=8)$ & $0(0 \%)(n=0)$ & 0.122 & $5(33.3 \%)(n=15)$ & $0(0 \%)(n=8)$ & 0.122 \\
\hline Total & $20(33.3 \%)(n=60)$ & $9(45 \%)(n=20)$ & $20(35.7 \%)(n=56)$ & $7(31.8 \%)(n=20)$ & 0.787 & $8(40 \%)(n=20)$ & $0(0 \%)(n=20)$ & $0.003^{*}$ \\
\hline
\end{tabular}

GABHS, group A beta hemolytic streptococcus.

*There was a significant difference in the total number of redundant cultures between the two groups.

Table 5. Antibiotic usage rate

\begin{tabular}{|c|c|c|c|c|c|c|c|c|}
\hline \multirow[b]{2}{*}{ Total score } & \multicolumn{5}{|c|}{ Number of patients on antibiotics } & \multicolumn{3}{|c|}{ Number of patients on unnecessary antibiotics } \\
\hline & $\begin{array}{c}\text { Group A } \\
(n=60)\end{array}$ & $\begin{array}{c}\text { Number of } \\
\text { positive GABHS }\end{array}$ & $\begin{array}{l}\text { Group B } \\
(n=56)\end{array}$ & $\begin{array}{c}\text { Number of } \\
\text { positive GABHS }\end{array}$ & $P$ value & Group A & Group B & $P$ value \\
\hline 2 & $21(100 \%)(n=21)$ & $0(0 \%)(n=4)$ & $13(65 \%)(n=20)$ & $2(22.2 \%)(n=9)$ & 0.0005 & $20(95.2 \%)(n=21)$ & $11(55.0 \%)(n=20)$ & $0.0005^{*}$ \\
\hline 3 & $10(76.9 \%)(n=13)$ & $2(25 \%)(n=8)$ & $7(50 \%)(n=14)$ & $5(45.5 \%)(n=11)$ & 0.148 & $8(61.5 \%)(n=13)$ & $2(14.3 \%)(n=14)$ & $0.011^{*}$ \\
\hline Total & $54(90.0 \%)(n=60)$ & $9(45 \%)(n=20)$ & $38(67.9 \%)(n=56)$ & $7(35 \%)(n=20)$ & 0.007 & $32(53.3 \%)(n=60)$ & $15(26.8 \%)(n=56)$ & $0.003^{*}$ \\
\hline
\end{tabular}

GABHS, group A beta hemolytic streptococcus.

*There were significant differences in the number of patients on unnecessary antibiotics between the two groups in respect to Scores 2 and 3 and the total number of patients.

was a significant drop of $26.5 \%(P=0.003)$ in the incidence of unnecessary antibiotic prescribing from Group A $(53.3 \%)$ to Group B (26.8\%). Antibiotic prescriptions were significantly lower in Group B $(67.9 \%, P=0.007)$ than in Group A $(90 \%, P=0.007)$ with a reduction of $22.1 \%$. Two patients from Group A were not initiated with an antibiotic despite of having positive culture results for GABHS, but such cases were absent in Group B. Table 5 shows the antibiotic usage rate against the total McIsaac score in the two groups.

The overall compliance rate significantly improved by 22.9\% $(P=0.0005)$ after the enforcement of the McIsaac rule. Clinicians' compliance was higher for moderate risk patients with a total score of $2(52.4 \%)$ in Group A, where this category of patients should be treated with an antibiotic based on the culture results only. For Group B, prescribers were $100 \%$ compliant in cases where the total score was 4 and 5, where patients should be treated with an antibiotic without any culture. The poorest compliance rate was seen in patients with Score 1 (in both groups). Although neither antibiotics nor culture swabs are recommended by the McIsaac rule for Score 1, all the patients (100\%) from Group A and three (50\%) from Group B, were given an antibiotic without supportive culture results. The clinicians were found to be compliant to the McIsaac rule in 38 patients (67.9\%) from Group B but just in 27 patients (45\%) from Group A. Table 6 details the prescribers' compliance to the McIsaac decision rule.

\section{DISCUSSION}

The McIsaac clinical decision rule was developed to improve the diagnosis of GABHS and the proper use of antibiotics in cases of sore throat. The rule's effectiveness was first identified when it was developed and evaluated by McIsaac and colleagues (13). In that study, the researchers reported a reduction of $48 \%$ in antibiotic use. Later, McIsaac and colleagues evaluated the performance of the rule in a new population (9), finding that it has reduced antibiotic prescriptions by $52.3 \%$ (9). This indicated that the McIsaac clinical decision rule is a reliable tool for better management of sore throat cases. Here in this report, we added to the current literature by evaluating this clinical decision rule in a tropical country (Malaysia) on the basis that GABHS has been reported to be common among culture-positive sore throat cases in tropical regions (14). Our findings proved that the McIsaac rule can be used efficiently to reduce the misuse of antibiotics and redundant sore throat cultures. The McIsaac rule was able to distinguish between viral and GABHS infections leading to sore throat on the basis of its scoring criteria. This can help to tackle the challenge of precise diagnosis of the etiology of sore throats, which will ultimately reduce bacterial resistance (15).

The mean age of both the groups was below $5 \mathrm{y}$, which is not uncommon since children aged below $5 \mathrm{y}$ are among the groups vulnerable to viral sore throat infections (16). There was significant difference in age distribution between the two groups. This may be due to local weather changes since Group 
Table 6. Prescribers' compliance with the Mclsaac decision rule

\begin{tabular}{lccl}
\hline Total score & $\begin{array}{c}\text { Number of compliant } \\
\text { cases from Group A }\end{array}$ & $\begin{array}{c}\text { Number of compliant } \\
\text { cases from Group B }\end{array}$ & $P$ value \\
\hline 1 & $0(0 \%)(n=4)$ & $3(50.0 \%)(n=6)$ & 0.076 \\
2 & $11(52.4 \%)(n=21)$ & $8(40.0 \%)(n=20)$ & $0.032^{*}$ \\
3 & $3(23.1 \%)(n=13)$ & $11(78.6 \%)(n=14)$ & $0.034^{*}$ \\
4 & $9(60 \%)(n=15)$ & $8(100 \%)(n=8)$ & 0.058 \\
5 & $4(57.1 \%)(n=7)$ & $8(100 \%)(n=8)$ & 0.077 \\
Total & $27(45 \%)(n=60)$ & $38(67.9 \%)(n=56)$ & $0.0005^{*}$ \\
\hline
\end{tabular}

*There were significant differences in the prescribers' compliance with the Mclsaac decision rule between the two groups in respect to Scores 2 and 3 and the total number of bed head tickets.

$B$ data were collected in the dry season and the transmission of viral URTIs increases in dry seasons in tropical regions. Most of the patients in our study had a score of 2, where the likelihood of GABHS is $10-20 \%$. Indeed, this was not unexpected since the etiology of most sore throat cases in children is viral infections (2-4). Thus, the Cochrane review's assertion regarding the lack of difference in complication rates between delayed and immediate antibiotic use in respiratory tract infections is justifiable (8). Delayed antibiotic use in the case of sore throat may not increase patients' risk of experiencing complications from GABHS, but will help to reduce the misuse of antibiotics. A decrease in the overuse of antibiotics is important in order to reduce bacterial resistance (9). The reduction of redundant sore throat swab cultures after the implementation of the McIsaac rule in our study was comparable with the findings of McIsaac and colleagues (13). In fact, sore throat swab cultures may not be the optimum diagnostic tool for GABHS infections because the accuracy of culture results is affected by several factors. Variations in collection, transportation, and culture methods are some of these factors (17-19). The overall antibiotic usage in our study was significantly reduced by using McIsaac rule. This further added to the inference that the McIsaac rule allows for more efficient and safe use of antibiotics.

The prescribers' compliance rate in our study was improved after the implementation of the McIsaac rule in the hospital. Nonetheless, our results imply that physicians are not totally adherent to the McIsaac clinical decision rule, although the improvement in adherence was more marked than in the findings of another study (20). Aaronson and colleagues had indicated a compliance rate of $15 \%$. In our study, there was a noticeable trend of performing unnecessary throat swab cultures and prescribing unnecessary antibiotics, despite the absence of clinical indication or without supportive culture results. A combination of physician-related factors, patientrelated factors, and facility-related factors may contribute to this state of affairs. The difficult discrimination between sore throat causes, and the fear of developing rheumatic fever, pressures physicians to prescribe antibiotics on a precautionary basis (21). In a study in India, physicians reported that they prescribe antibiotics even when not required, due to uncertainty regarding the cause of the sore throat. Besides, lack of time and overwhelming workload prevents the allocation of sufficient time to educate patients' parents about the consequences of antibiotic misuse. Accordingly, they tend to prescribe unnecessary antibiotics. The provider's personal experience and knowledge, particularly on antibiotic resistance, can also be a barrier to compliance with the McIsaac rule. In some cases, doctors tend to overuse antibiotics even for sore throats with a viral etiology, due to requests from the pharmacy department to clear drugs that are near expiry, including antibiotics (15). It has been reported that half of the prescriptions for antibiotics in a clinic in Malaysia were for URTIs (22). Referring to this large percentage, interventional awareness programs aim to increase the knowledge of physicians and pharmacists on the burden of bacterial resistance (22).

A further issue is that parental demand for irrational antibiotic prescriptions in the case of sore throat, particularly from the mother, has been found to be the primary reason for the overprescription of antibiotics (23). This behavior has been shown to be connected to low educational level (23). Macfarlane and colleagues conducted an observational study to assess the views and expectations of patients presenting with respiratory infections and found that $87 \%$ of patients believed that an antibiotic would help to solve the infection and $27 \%$ reported that they ask their physicians to prescribe antibiotics (24). In another survey, $43 \%$ felt that antibiotics can kill viruses and $8 \%$ disagreed with the potential for antibiotic resistance to evolve due to the overuse of antibiotics (25). Parental expectations regarding antibiotic prescriptions might be due to physicians' inaccurate perception of parents' attitudes, however (26). Stivers and colleagues found a disagreement between physicians' perceptions of parental expectations and parents' own reported expectations (26). Reduced awareness and social influences had a substantial impact on parental demands for antibiotics in another study $(23,25)$. Tailored awareness programs on bacterial resistance and the etiologies of sore throat infections are required for parents to create awareness about the consequences of overusing antibiotics. In addition to physician and parental factors, there are also facility-related factors which contribute to the reduced compliance to the McIsaac rule. The lack of rapid diagnostic kits to identify infections is one of these factors (25). In most of the health care facilities in Malaysia, culture results are only obtained $24 \mathrm{~h}$ after performing the throat swab or, in some cases, even longer. This may lead the physician to prescribe antibiotics empirically in fear of possible rheumatic fever complications. In this regard, physicians often neglect to take account of the fact that $90 \%$ of sore throat patients recover within $7 \mathrm{~d}$ without antibiotic use, regardless of sore throat etiology, while the rheumatic fever complications may only affect $3 \%$ of the infected cases (27).

It can be concluded that the McIsaac rule is an effective tool in reducing misuse of antibiotics and redundant throat swab cultures in children diagnosed with sore throat in Malaysia. There was a noticeable amelioration of $22.9 \%$ in terms of prescribers' compliance to the Malaysian Clinical Practice Guideline for sore throat after the enforcement of the McIsaac clinical decision rule in this study. We suggest performing more awareness programs targeted at health care providers 
and parents. This study was limited by the retrospective design which mitigated achieving equal severity of URTIs between the two groups. This may have interfered with the clinical decision of the prescribers.

\section{METHODS}

This was a retrospective study conducted in Hospital Kulim, Kedah, Malaysia. The study was done after the implementation of the McIsaac decision rule in the pediatric department in October 2012. We reviewed patient medical records for children who were initially diagnosed with sore throat between July 2012 to December 2012. Patients were excluded from the study if they were on antibiotics in the week before admission, if they were experiencing a recurrence of sore throat, if they were immunocompromised, or if they had underlying/a history of rheumatic fever. The total number of eligible bed head tickets included in the study was 116 . This was divided into two groups: Group A comprised bed head tickets before the implementation of the McIsaac decision rule (July to September 2012) and Group B comprised bed head tickets after the implementation of the rule (October to December 2012). Data on patients' demographics, medical history, concurrent medication, known allergies, clinical characteristics, throat swab cultures, and prescribed medications were collected. The McIsaac score was calculated based on patients' clinical information. When presenting the results, redundant throat swab culture refers to culture requests which did not comply with McIsaac's suggestions. Unnecessary antibiotic prescription refers to the situation where an antibiotic was given to a sore throat patient who did not actually need an antibiotic as per McIsaac's suggestions, or the antibiotic given was not the suitable or sensitive to the pathogen obtained from the culture results. The prescribers were considered compliant if they fulfilled the suggestions of the McIsaac rule for each score. If a prescriber failed to follow the suggestion for any McIsaac score, the action was considered as noncompliant.

The collected data were analyzed using the statistical package of social sciences software (IBM SPSS, version 20, SPSS, Chicago, IL). Descriptive statistics were used for the demographics and clinical data. The chi-square test or Fisher's exact test was used to compare between the two groups. This study was reviewed and approved by the Medical Research and Ethics Committee (MREC) of the Ministry of Health of Malaysia. Due to the retrospective nature of this study, the informed consent requirement was waived.

\section{ACKNOWLEDGMENTS}

We thank the Director General of Health Malaysia for the permission to publish this article. We acknowledge the efforts and the positive input of the Paediatric ward team at Hospital Kulim in the study.

\section{STATEMENT OF FINANCIAL SUPPORT}

The authors declare that no product was used in the study and that they had no financial assistance in support of this research.

Disclosure: The authors declare there are no conflicts of interest in this research.

\section{REFERENCES}

1. Schwartz B, Marcy SM, Phillips WR, Gerber MA, Dowell SF. Pharyngitis-principles of judicious use of antimicrobial agents. Pediatrics 1998;101:171-4.

2. Bisno AL, Gerber MA, Gwaltney JM, Kaplan EL, Schwartz RH. Practice guidelines for the diagnosis and management of group A streptococcal pharyngitis. Clin Infect Dis 2002;35:113-25.

3. Choby BA. Diagnosis and treatment of streptococcal pharyngitis. Am Fam Physician 2009;79:383-90.

4. Smeesters PR, Campos D Jr, Van Melderen L, de Aguiar E, Vanderpas J, Vergison A. Pharyngitis in low-resources settings: a pragmatic clinical approach to reduce unnecessary antibiotic use. Pediatrics 2006;118:e1607-11.
5. Martin JM, Green M, Barbadora KA, Wald ER. Group A streptococci among school-aged children: clinical characteristics and the carrier state. Pediatrics 2004;114:1212-9.

6. Steinhoff MC, Abd el Khalek MK, Khallaf N, et al. Effectiveness of clinical guidelines for the presumptive treatment of streptococcal pharyngitis in Egyptian children. Lancet 1997;350:918-21.

7. Van Howe RS, Kusnier LP 2nd. Diagnosis and management of pharyngitis in a pediatric population based on cost-effectiveness and projected health outcomes. Pediatrics 2006;117:609-19.

8. Spurling GK, Del Mar CB, Dooley L, Foxlee R, Farley R. Delayed antibiotics for respiratory infections. Cochrane Database Syst Rev 2013;4:CD004417.

9. McIsaac WJ, Goel V, To T, Permaul JA, Low DE. Effect on antibiotic prescribing of repeated clinical prompts to use a sore throat score. J Fam Pract 2002;51:339-44.

10. Wise R, Hart T, Cars $\mathrm{O}$, et al. Antimicrobial resistance. Is a major threat to public health. BMJ 1998;317:609-10.

11. World Health Organization. The Management of Acute Respiratory Infections in Children: Practical Guidelines for Outpatient Care. Geneva, Switzerland: World Health Organization, 1995. (http://www.who.int/iris/ handle/10665/41803.)

12. Rimoin AW, Hamza HS, Vince A, et al. Evaluation of the WHO clinical decision rule for streptococcal pharyngitis. Arch Dis Child 2005;90: 1066-70.

13. McIsaac WJ, White D, Tannenbaum D, Low DE. A clinical score to reduce unnecessary antibiotic use in patients with sore throat. CMAJ 1998;158: 75-83.

14. Steer AC, Jenney AW, Kado J, et al. Prospective surveillance of streptococcal sore throat in a tropical country. Pediatr Infect Dis J 2009;28:477-82.

15. Diaz MC, Symons N, Ramundo ML, Christopher NC. Effect of a standardized pharyngitis treatment protocol on use of antibiotics in a pediatric emergency department. Arch Pediatr Adolesc Med 2004;158:977-81.

16. Icahn School of Medicine at Mount Sinai. Influenza (Flu). Mount Sinai, 2016. (http://www.mountsinai.org/patient-care/health-library/diseasesand-conditions/influenza.) Accessed 28 February 2016.

17. Ministry of Health Malaysia. Clinical Practice Guidelines on Management of Sore Throat. Kuala Lumpur, Malaysia: Ministry of Health Malaysia, 2003.

18. Kaplan E. The throat culture: its techniques, pitfalls, limitations and meaning. Conn Med 1973;37:45-8.

19. Woolley SL, Bernstein JM, Davidson JA, Smith DR. Sore throat in adultsdoes the introduction of a clinical scoring system improve the management of these patients in a secondary care setting? J Laryngol Otol 2005;119:550-5.

20. Aaronson E, Ludwig N, Price I. Pharyngitis in the emergency department: an evaluation of the McIsaac clinical decision rule in practice. MUMJ 2011;8:16-9.

21. Kotwani A, Wattal C, Katewa S, Joshi PC, Holloway K. Factors influencing primary care physicians to prescribe antibiotics in Delhi India. Fam Pract 2010;27:684-90.

22. Teng CL, Achike FI, Phua KL, et al. General and URTI-specific antibiotic prescription rates in a Malaysian primary care setting. Int J Antimicrob Agents 2004;24:496-501.

23. Sadoh WE, Sadoh AE, Eki-Udoko FE. Parental contribution to over prescription of antibiotics for sore throat in children. Niger J Paediatr 2015;42:98-102.

24. Macfarlane J, Holmes W, Macfarlane R, Britten N. Influence of patients' expectations on antibiotic management of acute lower respiratory tract illness in general practice: questionnaire study. BMJ 1997;315:1211-4.

25. Hosey MC. Antibiotics in Primary Care: Factors Leading to Inappropriate Prescribing. Pullman, WA: Washington State University, 2012.

26. Stivers T, Mangione-Smith R, Elliott MN, McDonald L, Heritage J. Why do physicians think parents expect antibiotics? What parents report vs what physicians believe. J Fam Pract 2003;52:140-8.

27. Richardson K. Non-antibiotic treatment sore throat. Emerg Nurse 2011;18:12-6. 